\title{
Multidisciplinary Care
}

\section{Pharmacist-Led, Interdisciplinary Model for Delivery of Supportive Care in the Ambulatory Cancer Clinic Setting}

\author{
By John Valgus, PharmD, BCOP, CPP, Sandra Jarr, RN, MSN, Robert Schwartz, MA, Michelle Rice, RN, MSN, \\ and Stephen A. Bernard, MD
}

University of North Carolina (UNC) Hospitals; UNC Eshelman School of Pharmacy; UNC School of Medicine; Sheps Center for Health Services Research, Chapel Hill; Duke University School of Nursing, Durham, NC

\begin{abstract}
Purpose: To describe a pharmacist-led, interdisciplinary method of care delivery begun at the University of North Carolina. We describe the characteristics of the population seen and the role of the individual members of the interdisciplinary team, and provide an early analysis of the program's impact on symptom improvement.
\end{abstract}

Methods: A supportive care consultation service was begun at the University of North Carolina Hospitals to serve adult outpatients with cancer undergoing treatment or follow-up. Patients data were entered into an institutional review board-approved database to permit detailed assessments over time. Patient demographics were analyzed using descriptive statistics, medications used and changes made were noted, and symptom scores from a previously described instrument were captured and compared over time.

\section{Introduction}

Palliative care in North America has increasingly been delivered in the outpatient setting, using an interdisciplinary approach to care for patients with symptoms resulting from cancer or its treatment. ${ }^{1}$ Parallel with the development of outpatient palliative care services, there has been an increased integration of the pharmacist into the health care team. ${ }^{2-4}$ This integration initially occurred in the inpatient setting, where the complexities of medications-with increasing numbers of drugs and the potential for drug interactions - necessitated the presence of a pharmacist on the health care team. Use of pharmacists in the ambulatory setting, specifically in oncology supportive care, has been rarely reported. 5

The University of North Carolina (UNC) Hospitals inpatient Palliative Care Consult Service was begun in 2002 to provide treatment to patients with cancer and other lifelimiting illnesses. In February 2008, funding was received to begin an outpatient supportive care consultation service in the adult cancer clinics: radiation oncology, surgical oncology, gynecologic oncology, hematology, and medical oncology. This consultative service uses a roving pharmacist/nurse model in coordination with an oncology/palliative care physician and is the subject of this report.
Results: Patients were seen from all adult oncology services, including gynecologic, radiation, medical, and surgical. The characteristics of the population seen were similar to those of the hospital population as a whole. Most of the patients were seen for pain management, and many required a medication change. Symptom scores improved by the second visit, and the improvement was maintained.

Conclusion: We are able to demonstrate that the use of a pharmacist-led, interdisciplinary team produced an improvement in symptom scores comparable to what has been seen in the inpatient palliative care service within our institution. Projected shortages of oncology providers may be mitigated by pharmacists working in collaborative practices, with prescriptive authority, in the ambulatory oncology setting.

\section{Methods}

The Supportive Care Consult Service and Clinic includes an oncology pharmacy residency-trained, board-certified oncology pharmacist. The pharmacist joined the practice part-time in February 2008 and became full-time in December 2008. The pharmacist works as a clinical pharmacist practitioner, a licensed pharmacist approved to provide drug therapy management, including administration of controlled substances, under the direction of a licensed physician. Only pharmacists approved by the North Carolina Pharmacy Board and Medical Board may legally identify themselves as a clinical pharmacist practitioner. The practice is based on evidence-based treatment algorithms for commonly seen symptoms such as pain, nausea and vomiting, and constipation. The second member of the team is an advanced practice nurse (clinical nurse specialist) with a background in intensive care unit nursing and nursing administration, who had completed a sabbatical in palliative care at the Northwestern Memorial Hospital Palliative Care Program. The third member of the team is a senior physician who is a board-certified medical oncologist and hospice and palliative medicine specialist, who had trained in palliative medicine at the University of Alberta. Funding for this service is provided by the UNC School of Medicine (physician), UNC Hospital (nurse and pharmacist, partial), UNC School of Pharmacy (pharmacist, partial), and a grant from the UNC 
Lineberger Comprehensive Cancer Center University Cancer Research Fund (pharmacist, partial).

The clinical information on which the present analysis is based is taken from two sources: an institutional review boardapproved prospective database (demographics, symptom scores) and a retrospective chart review (medications for symptoms, substance abuse, and insurance). Time intervals used for this report are February 1, 2008 through July 31, 2009 (prospective database) and February 1, 2008 through January 31, 2009 (retrospective database). The latter interval permitted a more detailed analysis of the patient population, which was not available from the prospective database at the time of analysis.

Symptom scores were assessed for each visit using an instrument developed for the Palliative Care Consult Service that comprises 11 symptom-based items scored on a 5-point Likert-type scale $(5=$ most severe $)$ and is adapted from the revised McCorkle Symptom Distress Scale. ${ }^{6}$ We omitted symptoms not seen in an ambulatory outpatient population, leaving eight symptoms: pain, shortness of breath, nausea, mood, constipation, insomnia, delirium, and fatigue. Scores were entered into the prospective database and analyzed to assess symptom improvement among patients seen in the Supportive Care Consult Service and Clinic.

The consultation service is available Monday through Friday during clinic hours. Initial requests for consultation are called in to the nurse, who then triages the consult to one or more of the providers. A novel feature of the service is the pharmacist and nurse going as a team to the clinic where the patient was being seen for their oncologic care, with physician involvement as needed. This model avoided an additional clinic visit for the patients, most of whom live an hour or more away from the institution. Approval for the consult was ultimately given by the primary oncologist, and all recommendations were conveyed to this individual. This requirement served to ensure that the consult was sanctioned by the person most knowledgeable of the patient's current status, and it made the primary oncologist an active participant in the conclusions and recommendations.

In April 2008, the need for a more structured visit for patients with complex care issues or need for ongoing follow-up led to the establishment of a half-day clinic. Patients are seen initially by the pharmacist; a limited cognitive assessment is carried out together with a detailed medication history. The nurse and physician then see the patient and complete a symptom management assessment. A team meeting is then held, after which the treatment recommendations are presented to the patient and family; they are then implemented with any further modifications. In addition to medication changes and direct symptom interventions, referrals to other supportive care team members-clinical dietician, psychooncologist, and anesthesia pain service—may also occur.

\section{Results}

Table 1 shows the demographic characterisics of the patients seen. A slight preponderance of women was seen, nearly half the patients were older than 60 years of age, and one fifth of the patients were younger than the age of 44 . One fifth of the patients were African American (slightly less than the institutional proportion). Eight percent were of Hispanic origin, and one patient was Native Amer-
Table 1. Patient Demographics, Supportive Care Consult Service and Clinic, University of North Carolina Hospitals ( $N=89$, Feb 1, 2008-July 31, 2009)

\begin{tabular}{|c|c|c|}
\hline Characteristic & No. & $\%$ \\
\hline \multicolumn{3}{|l|}{ Sex } \\
\hline Male & 42 & 47 \\
\hline Female & 47 & 53 \\
\hline \multicolumn{3}{|l|}{ Age, years } \\
\hline$<44$ & 40 & 21 \\
\hline $44-59$ & 30 & 34 \\
\hline$>60$ & 19 & 45 \\
\hline \multicolumn{3}{|l|}{ Race/ethnicity } \\
\hline White & 62 & 70 \\
\hline African American & 19 & 21 \\
\hline Hispanic & 7 & 8 \\
\hline Native American & 1 & 1 \\
\hline \multicolumn{3}{|c|}{ Proportion of patients seen by tumor type } \\
\hline Lung & 18 & 20 \\
\hline Gynecologic & 18 & 20 \\
\hline Head and neck & 14 & 16 \\
\hline Genitourinary & 11 & 11 \\
\hline Gl & 7 & 8 \\
\hline Breast & 6 & 7 \\
\hline Hematologic & 1 & 1 \\
\hline Other & 14 & 16 \\
\hline \multicolumn{3}{|l|}{ Sources of referral } \\
\hline Radiation oncology & 43 & 48 \\
\hline Medical oncology & 29 & 33 \\
\hline Gynecologic oncology & 10 & 11 \\
\hline Surgical oncology & 7 & 8 \\
\hline \multicolumn{3}{|c|}{ Reason for consult (primary symptom) } \\
\hline Pain & 88 & 75 \\
\hline Constipation & 13 & 11 \\
\hline Nausea or vomiting & 10 & 8 \\
\hline Anxiety/mood & 5 & 4 \\
\hline Spiritual & 2 & 2 \\
\hline
\end{tabular}

NOTE: Total No. of patients with each symptom will equal $>89$ because multiple patients list with more than one primary reason for consult.

ican. Nearly one half of the patients were referred from the radiation oncology clinics, with approximately $20 \%$ coming from either surgical oncology or gynecologic oncology; the remainder were from medical oncology. Except for patients undergoing bone marrow transplantation, patients with all tumor categories were seen. Predominately, the patients had lung cancer or gynecologic cancer; however, less common tumor types such as sarcoma were also represented in the diagnostic categories. Three quarters of the consultations were for pain management. Other symptoms included constipation, nausea or vomiting, and anxiety.

Table 2 shows the individual practitioner involvement in patient referrals. In the first 18 months of the service, 89 new patients were seen. Overall there were 292 patient/provider encounters, reflecting both new and return visits. Approximately two thirds of the visits were either with the nurse, pharmacist, or 
Table 2. Encounters by Practitioner

\begin{tabular}{ll}
\hline $\begin{array}{l}\text { Practitioners Consulted } \\
\text { per Encounter }\end{array}$ & $\begin{array}{l}\text { Percentage of Encounters } \\
(\mathbf{N}=\mathbf{2 9 2})\end{array}$ \\
\hline Nurse alone & 17.6 \\
Physician alone & 1.4 \\
Pharmacist alone & 10.3 \\
Nurse and pharmacist & 28.6 \\
Nurse and physician & 13.1 \\
Physician and pharmacist & 5.2 \\
\hline Nurse, physician, and pharmacist & 22.7 \\
\hline
\end{tabular}

both members of the team. The physician was present for $40 \%$ of the visits; many of these occurring with the opening of the clinic. Pharmacist involvement in patient encounters nearly doubled (from $43 \%$ to $77 \%$ ) after the pharmacist joined the practice fulltime in December 2008, reflecting the difference between a fulltime commitment and a voluntary effort.

Seventy-two percent of the patients seen in consultation were receiving active treatment, and $28 \%$ were not receiving active treatment, either because treatment was no longer felt to be beneficial or because patients were cancer free and were being seen for management of symptoms that had resulted from treatment. Because many of the referrals were through the radiation oncology clinics, more than half the patients had been treated with radiation, with $40 \%$ having received chemotherapy and $5 \%$ having combined chemoradiotherapy. Ten percent of the patients were self-pay, $44 \%$ had private insurance, and $46 \%$ had Medicare or Medicaid. From the review of the 54 patients seen in the first year, we also looked at the medications that the patients were receiving. Fifty-two of the 54 were being administered some form of pain medication, with $17 \%$ receiving methadone and $74 \%$ receiving other long-acting opioids, including morphine, oxycodone, and transdermal fentanyl. After the initial visit, $40 \%$ of the patients had an increase in their medication dose, $23 \%$ had a new medication added, $15 \%$ were switched to another opioid, and 15\% had no change. Those who were switched or had a new medication added most often received methadone as the new medication.

During the first 18 months of the service, the volume of new patients increased from 4.5 per month to 6.6 , with the number of encounters going from 13 to 20 per month. Initial analysis of the first 49 patients seen who were entered in the research database showed improvements in all symptom scores, including pain, nausea, and constipation, by the second visit. (Figure 1). On average, patients were seen three times. The reduction in symptom scores was maintained in the third visit and beyond; however, the number of patients assessed became increasingly lower after three visits.

\section{Discussion}

In a recent $\mathrm{ASCO}$ position paper that reviewed the need for integration of palliative care into oncology, Ferris et $\mathrm{al}^{7}$ describe the gradual expansion of the role of palliative care in cancer treatment over the last 1 to 2 decades and discuss the next steps in this process. Increasingly, oncologic care has shifted to the outpatient setting. Palliative care, although initially largely an

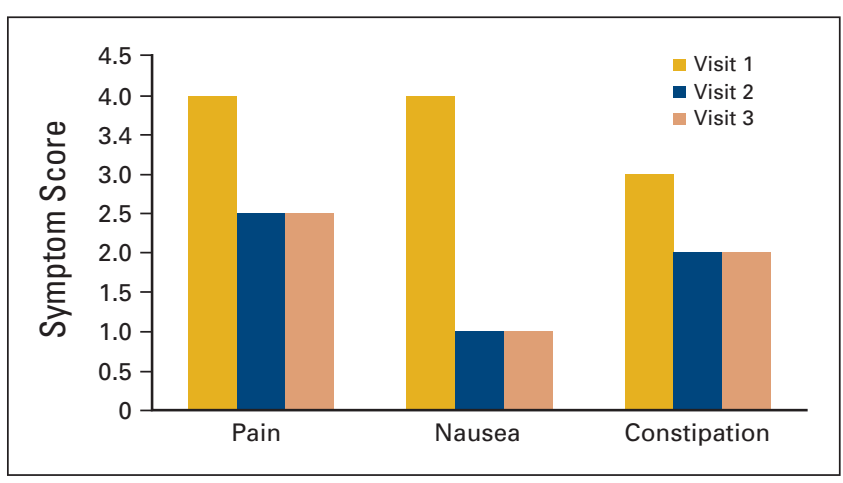

Figure 1. Change in symptom scores over three visits.

inpatient specialty, has also begun to increase its presence in the outpatient setting. A recent survey of 71 National Cancer Institute (NCI) -designated cancer centers and 71 non-NCI-designated centers showed that an outpatient palliative care clinic was incorporated into $59 \%$ and $22 \%$, respectively, of the sites surveyed. ${ }^{8}$ Bruera, an author of the position paper for ASCO, has described such a successful interdisciplinary clinic model. ${ }^{1}$ Pituskin has described a multidisciplinary outpatient clinic for patients receiving palliative radiotherapy. ${ }^{9}$

A recent survey of 12 leading academic outpatient palliative care programs by Rabow et $\mathrm{al}^{10}$ highlights the characteristics of these programs. Anywhere from 90 to 1,400 patients are seen annually, with 48 to 840 of these being new patients. Staffing was reported as including physicians in $91 \%$ of the programs, advanced practice nurses or nurse practitioners in 55\%, and social workers in $45 \%$. Rabow et al do not comment on the presence of pharmacists in the supportive care setting. Together with the shift of palliative care and oncologic care to the outpatient setting, there is increasing recognition by the medical community of the role for a clinical pharmacist in the ambulatory setting. The increase in the number of states that have collaborative drug therapy management legislation and the development of collaborative practice models have allowed pharmacists to play a greater role in the care of patients with increasingly complex medication needs. ${ }^{2,5,11-15}$ Pharmacists working within collaborative practice agreements with physicians have been endorsed by the American College of Physicians-American Society of Internal Medicine. ${ }^{16}$

A recent Cochrane review of 25 studies has indicated the successful integration of pharmacists into the outpatient setting, with a positive impact on number of inpatient admissions, nonscheduled health services, and the costs of drugs. However, measures of quality of life for patients did not change. ${ }^{3}$

Atayee et $\mathrm{al}^{5}$ describe the use of a pharmacist practice model for palliative care in the ambulatory setting. The pharmacist was based in a retail pharmacy but functioned as a member of the palliative care team in the outpatient setting and had prescriptive powers authorized by the State of California. Bruera et $\mathrm{al}^{1}$ describe the use of a pharmacist and a nurse in a palliative care clinic. Recommendations were made $87 \%$ and $89 \%$ of the time by these two providers, respectively. Our recommendation percentages are similar to these described by Bruera et al. 
We believe that the outpatient supportive care service that we have described has several novel features: The pharmacist is an integral and essential member of the team and serves as the main provider of supportive care services for many of these patients. Through the use of a collaborative practice agreement (initially established by legislation in North Carolina in 199915), the pharmacist is able to prescribe both opioids and other medications. The movement of oncologic and palliative care into the outpatient setting, the development of collaborative drug therapy models for delivery of care, and the increasing use of clinical pharmacy services in the outpatient setting are trends that have come together in the service that we have developed. The use of a roving pharmacist/ nurse model has not been discussed in other reports of ambulatory supportive care (or palliative care) clinics or consultative services ${ }^{1,8,10,17}$ This consultative service permits the primary oncologist to focus on specific oncology-related issues, while continuing to involve this individual in supportive care decisions.

The most recent ASCO report on the oncology workforce predicts significant oncologist shortages by the year $2020 .{ }^{18}$ The shortage estimate of 2,350 to 3,800 oncologists represents up to a third of the total number of oncologists available in 2005. The report addressed the use of nurse practitioners and physician assistants to help improve practice efficiency of oncologists but did not mention the possible role of the clinical pharmacist. Use of qualified clinical pharmacists working in collaborative practice agreements similar to other physician extenders is another possible solution to help bridge the gap between the demand for oncology services and the projected shortage of oncology practitioners in the United States in the near future.

In our practice, the physician was directly involved in approximately $42 \%$ of patient encounters, although all encounters were routinely reviewed by the physician. This was largely due to the provider privileges and prescriptive authority of the clinical pharmacist practitioner, which enabled the team to

\section{References}

1. Bruera E, Michaud M, Vigano A, et al: Multidisciplinary symptom control clinic in a cancer center: A retrospective study. Support Care Cancer 9:162-168, 2001

2. Abramowitz PW: The evolution and metamorphosis of the pharmacy practice model. Am J Health Syst Pharm 66:1437-1446, 2009

3. Beney J, Bero LA, Bond C: Expanding the roles of outpatient pharmacists: Effects on health services utilisation, costs, and patient outcomes. Cochrane Database Syst Rev CD000336, 2000

4. Kuo GM, Buckley TE, Fitzsimmons DS, et al: Collaborative drug therapy management services and reimbursement in a family medicine clinic. Am J Health Syst Pharm 61:343-354, 2004

5. Atayee RS, Best BM, Daniels CE: Development of an ambulatory palliative care pharmacist practice. J Palliat Med 11:1077-1082, 2008

6. Bernard S, Lee C, Usher B, et al: Symptom assessment tools used in a hospital-based pain and symptom care program at the University of North Carolina: A novel proxy rating. Proc Am Soc Clin Oncol 24: 487s, 2006 (abstr 8579)

7. Ferris FD, Bruera E, Cherny N, et al: Palliative cancer care a decade later: Accomplishments, the need, next steps-From the American Society of Clinical Oncology. J Clin Oncol 27:3052-3058, 2009

8. Hui D, Elsayem A, De la Cruz M, et al: Availability and integration of palliative care at US cancer centers. JAMA 303:1054-1061, 2010

9. Pituskin E, Fairchild A, Dutka J, et al: Multidisciplinary team contributions within a dedicated outpatient palliative radiotherapy clinic: A prospective descriptive study. Int J Radiat Oncol Biol Phys [epub ahead of print on January 25, 2010] manage supportive care issues according to established collaborative practice agreements without the physician present. Unfortunately, pharmacists are not currently recognized as providers by most payers, and billing by the pharmacist was only accomplished by a facility fee billing model. Although this model can support the pharmacist's salary, recognition of pharmacists as providers will be crucial to allow similar models of practice to be developed in non-hospital-based clinic settings.

\section{Accepted for publication on September 9, 2010.}

\section{Acknowledgment}

Supported in part by a grant from the Lineberger Comprehensive Cancer Center University Cancer Research Fund. Presented in part at the Annual Meeting of the American Society of Clinical Oncology, Chicago, June 2010 (abstract \#51146).

Authors' Disclosures of Potential Conflicts of Interest

The authors indicated no potential conflicts of interest.

\section{Author Contributions}

Conception and design: John Valgus, Stephen A. Bernard

Provision of study materials or patients: John Valgus, Sandra Jarr, Stephen A. Bernard

Collection and assembly of data: John Valgus, Sandra Jarr, Michelle Rice, Stephen A. Bernard

Data analysis and interpretation: John Valgus, Robert Schwartz, Michelle Rice, Stephen A. Bernard

Manuscript writing: John Valgus, Sandra Jarr, Stephen A. Bernard

Final approval of manuscript: John Valgus, Sandra Jarr, Robert Schwartz, Michelle Rice, Stephen A. Bernard

Corresponding author: John Valgus, PharmD, BCOP, CPP, Department of Pharmacy, University of North Carolina, CB\#7600, 101 Manning Dr, Chapel Hill, NC 27514; e-mail: JValgus@unch.unc.edu.

\section{DOI: 10.1200/JOP.2010.000033}

10. Rabow MW, Smith AK, Braun JL, et al: Outpatient palliative care practices. Arch Intern Med 170:654-655, 2010

11. Anaya JP, Rivera JO, Lawson K, et al: Evaluation of pharmacist-managed diabetes mellitus under a collaborative drug therapy agreement. Am J Health Syst Pharm 65:1841-1845, 2008

12. Carmichael JM, O'Connell MB, Devine B, et al: Collaborative drug therapy management by pharmacists. American College of Clinical Pharmacy. Pharmacotherapy 17:1050-1061, 1997

13. Hammond RW, Schwartz AH, Campbell MJ, et al: Collaborative drug therapy management by pharmacists-2003. Pharmacotherapy 23:1210-1225, 2003

14. Landis NT: Panelists describe collaborative drug therapy management efforts. Am J Health Syst Pharm 53:501-502, 1996

15. Landis NT: North Carolina, Louisiana legislate collaborative drug therapy management Am J Health Syst Pharm 56:2019, 1999

16. Keely JL: Pharmacist scope of practice. Ann Intern Med 136:79-85, 2002

17. Fairchild A, Pituskin E, Rose B, et al: The rapid access palliative radiotherapy program: Blueprint for initiation of a one-stop multidisciplinary bone metastases clinic. Support Care Cancer 17:163-170, 2009

18. Erikson C, Salsberg E, Forte G, et al: Future supply and demand for oncologists: Challenges to assuring access to oncology services. J Oncol Pract 3:79-86, 2007 